\title{
HD 65949: the highest known mercury excess of any CP star?
}

\author{
C. R. Cowley ${ }^{1}$, S. Hubrig ${ }^{2}{ }^{\star}$, G. F. González ${ }^{3}$, and N. Nuñez ${ }^{4}$ \\ 1 Department of Astronomy, University of Michigan, Ann Arbor, MI 48109-1090, USA \\ e-mail: cowley@umich.edu \\ 2 European Southern Observatory, Casilla 19001, Santiago 19, Chile \\ e-mail: shubrig@eso.org \\ 3 Complejo Astronómico El Leoncito, Casilla 467, 5400 San Juan, Argentina \\ e-mail: fgonzalez@casleo.gov.ar \\ 4 Departamento de Geofísica y Astronomía, Facultad de Ciencias Exactas Físicas y Naturales, Universidad Nacional de San Juan, \\ Argentina
}

Received 10 June 2006 / Accepted 29 June 2006

\section{ABSTRACT}

\begin{abstract}
ESO spectra of HD 65949 show it to be unlike any of the well-known types within its temperature range $\approx 13600 \mathrm{~K}$. It is neither a silicon, nor a mercury-manganese star, though it has a huge $\mathrm{Hg}$ II line at $\lambda 3984$. We estimate $\log (\mathrm{Hg} / \mathrm{H})+12.0 \approx 7.4$. This is higher than any published stellar mercury abundance. HD 65949 is a member of a nearby open cluster, NGC 2516, which is only slightly older than the Pleiades, and has been of recent interest because of its numerous X-ray emission stars, including HD 65949 itself, or a close companion. A longitudinal magnetic field of the order of -290 Gauss at the $4.7 \sigma$ level was very recently diagnosed from accurate circular spectropolarimetric observations with FORS 1 at the VLT.

The spectral lines are sharp, allowing a thorough identification study. Second spectra of Ti, Cr, and Fe are rich. Mn II is well identified but not unusually strong. Numerous lines of S II and P II are found, but not Ga II. The resonance lines of Sr II are strong. While many Y II lines are identified, and $\mathrm{Nb}$ II is very likely present, no Zr II lines were found. Xe II is well identified. Strong absorptions from the third spectra of the lanthanides Pr, $\mathrm{Nd}$, and Ho are present, but lines from the second spectra of lanthanides are extremely weak or absent. Among lines from the heavier elements, those of Pt II are clearly present, and the heaviest isotope, ${ }^{198} \mathrm{Pt}$, is indicated. The uncommon spectrum of Re II is certain, while Os II and Te II are highly probable. Several of the noted anomalies are unusual for a star as hot as HD 65949.
\end{abstract}

Key words. stars: chemically peculiar - stars: abundances - stars: individual: HD 65949

\section{Introduction}

HD 65949 may have the highest mercury abundance of any known star. The unusual strength of $\mathrm{Hg}$ II $\lambda 3984$ was already noted by Abt \& Morgan (1969) in their study of the open cluster NGC 2516. They did not call it a manganese star.

The age of the cluster was estimated as $\log t=8.2 \pm 0.1$ (Sung et al. 2002). Several studies indicate an X-ray source closely coincident with the position of HD 65949 (cf. Damiani et al. 2003, Table 4, Source No. 44).

Chemically peculiar $(\mathrm{CP})$ stars in the temperature range corresponding to mid-B to A0 are usually either silicon or mercurymanganese stars. The former belong to the magnetic sequence, designated CP2 by Preston (1974).

Line identifications for HD 65949 were made with the help of predicted line strengths from a model atmosphere with $T_{\text {eff }}=$ $13600 \mathrm{~K}$ and $\log (g)=4.0$, derived from Strömgren colors and $\mathrm{H} \beta$ photometry (cf. Moon 1984; Moon \& Dworetsky 1985). Artificially high abundances of "metals" were assumed in order to avoid missing lines. Central intensities greater than 0.001 were derived for all lines $(\lambda \lambda 3500-9400)$ in VALD (Kupka et al. 2000). The technique was presented by Cowley (1995) and is similar to one available to users of VALD.

A few abundances are derived below. These are based on a provisional or temporary model with the same $T_{\text {eff }}$ and $\log (g)$

\footnotetext{
* Based on ESO programe 076.D-0172(A).
}

from Strömgren photometry, but with abundances enhanced by a factor of 3 for atomic numbers $(Z)$ from 3 to 35 , a factor of 10 for $Z$ from 36 to 72 , and 100 for $Z$ above 73. Following Dworetsky (2005), we assumed a depleted helium abundance, $\mathrm{He} / \mathrm{H}=0.0085$.

We have placed a detailed line identification list with additional references online: http://www.astro.1sa.umich. edu/cowley/hd65949. We also provide links to two tables, highlighting the Pt II and Re II spectra.

\section{Observations}

Spectra of HD 65949 were obtained on 4 consecutive nights, from 20 to 23 October 2005, with the FEROS echelle spectrograph on the $2.2 \mathrm{~m}$ telescope at La Silla. Basic steps of spectroscopic reduction (bias subtraction, division by a normalized flat field, extraction of a 1D spectrum, continuum normalization and wavelength calibration) were based on IRAF.

The wavelength coverage is from 3530 to $9220 \AA$, and has a nominal resolving power of 48000 , with a $\mathrm{S} / \mathrm{N}$ ratio of ca. 200-250. The spectra were mildly Fourier filtered before the wavelengths were measured at Michigan.

A single longitudinal magnetic field measurement in HD 65949 was recently carried out by Hubrig et al. (2006) using FORS 1 at the VLT in its spectropolarimetric mode. A weak 


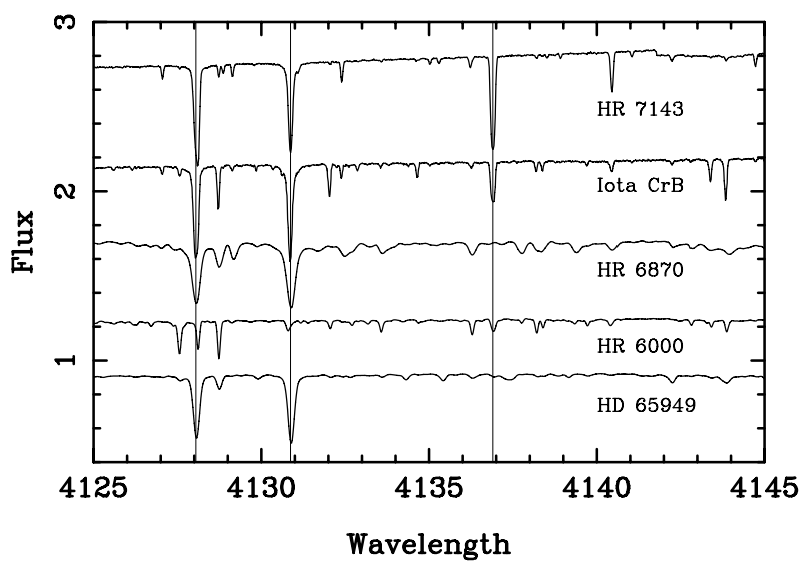

Fig. 1. Thin vertical lines are at the wavelengths of the strong Si II doublet, $\lambda \lambda 4128$ and 4130 , and Mn II $\lambda 4137$, which appear as a "triplet" in the upper two classical HgMn stars. The lower three stars are neither HgMn nor silicon (CP2) stars (cf. Sect. 6). Note the weakness of the Si II lines in HR 6000, and the Mn II line in HR 6870 and HR 65949. The absorption features are not precisely bisected because of significant blending.

magnetic field of the order -290 Gauss at the $4.7 \sigma$ level was detected.

\section{Manganese, mercury, and platinum}

At higher dispersion, HgMn stars may be recognized by the presence of Mn II 24137 , which forms an easily recognizable triplet with the nearby Si II doublet. This triplet is illustrated in Fig. 1. It is readily seen for the star labeled NGC $2516 \mathrm{~B}$ on Plate 17 of the atlas by Morgan et al. (1978), based on $39 \AA / \mathrm{mm}$ spectrograms. The Mn II line does not appear in the Multiplet Tables (Moore 1945).

One sees immediately from Fig. 1 that HD 65949 cannot be classified as a manganese star. The strong Mn II line $\lambda 4137$ is at best marginally present. Mn II, $\lambda 4137$ is somewhat weaker in the cooler HgMn star $\iota \mathrm{CrB}$ than in the hotter HR 7143. Generally, though not always, the Mn II spectrum strengthens toward the hotter HgMn types, reaching a maximum near $14000 \mathrm{~K}$. Thus HD 65949 is near the temperature where Mn II would be expected to reach a maximum.

The Hg II line 13984 , however, has a huge equivalent width (cf. Fig. 2). With one possible exception, it is larger than any of the values listed for the 43 stars included in the studies of White et al. (1976), or Woolf \& Lambert (1999). The possible exception is the value Woolf and Lambert give, $177 \mathrm{~m} \AA$ for HR 2844. They note that White et al. give a smaller value: $W_{\lambda}=144 \mathrm{m \AA}$. The star has substantial rotational broadening, $v \cdot \sin (i) \approx 30$, making an accurate measurement of the equivalent width difficult.

We estimate $W_{\lambda}(3984) \approx 175 \mathrm{~m} \AA$, using the wing of $\mathrm{H} \epsilon$ as the (local) continuum, and excluding the nearby absorption in the red wing (cf. Fig. 2). This line has a distinct minimum, at $3984.23 \pm 0.02 \AA$, too long for it to be ${ }^{204} \mathrm{Hg}$ II, 13984.07 (We adopt $\mathrm{Hg}$ isotopic wavelengths from Woolf and Lambert). Our measured wavelength for the minimum of the strong feature is $3984.01 \pm 0.01 \AA$, indicating that ${ }^{204} \mathrm{Hg}$ must be a major contributor to the strong absorption - it is not the weaker absorption on the red wing. A calculated profile based on $(1 / 6)^{200} \mathrm{Hg}$, $(1 / 2)^{202} \mathrm{Hg},(1 / 3)^{204} \mathrm{Hg}$, and ignoring other isotopic contributions fit the profile reasonably well.

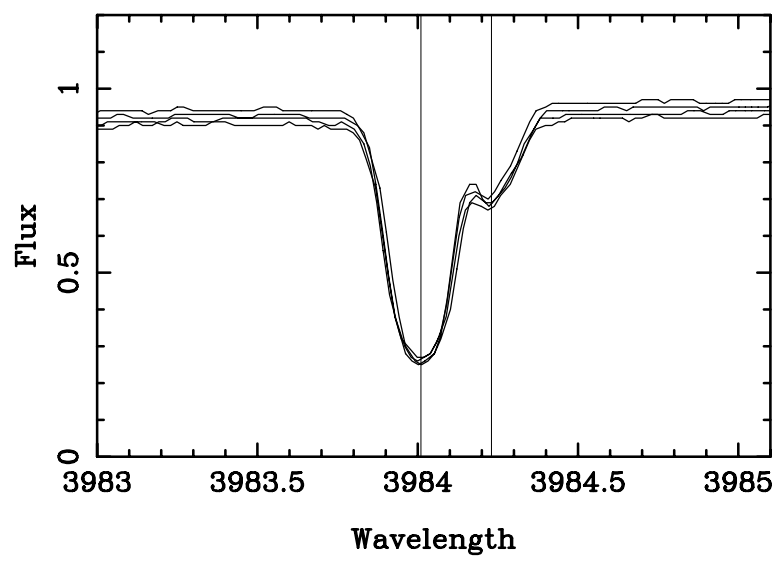

Fig. 2. Profiles of the $\lambda 3984$-region obtained on 4 sequential nights. They have been shifted to bring the mean wavelength of the strong component to $3984.01 \AA$. Adopted continuua from the original reductions have not been modified. The feature at $3984.23 \AA$, is primarily due to Re II (see text).

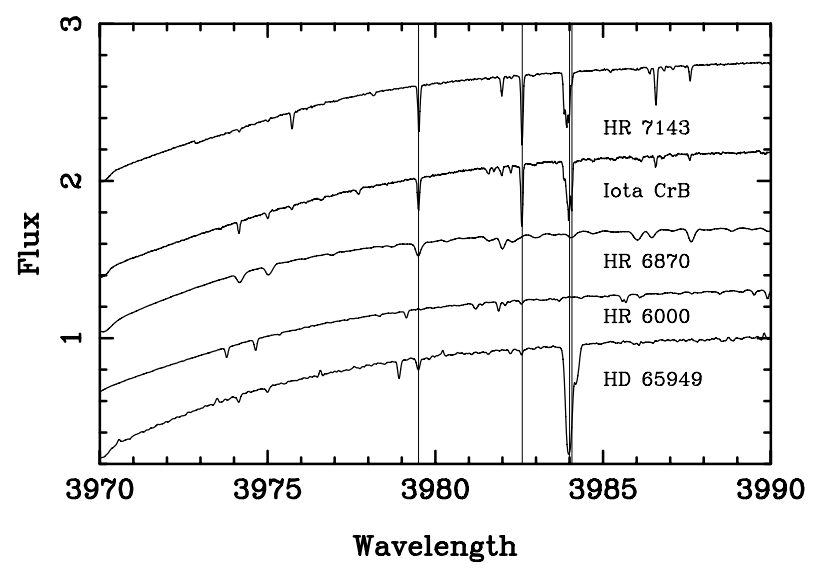

Fig. 3. Thin vertical lines are at the wavelengths of ${ }^{204} \mathrm{Hg}$ II, $\lambda \lambda 3984.07$; ${ }^{200} \mathrm{Hg}$ II, 3983.91; 3982.59 (Y II-6), and 3879.50 (Cr II-183).

Table 1. Provisional Abundances from Hg I and II.

\begin{tabular}{lcccc}
\hline \hline Spectrum & $\lambda($ VALD $)$ & $\lambda(\mathrm{HD} 65949)$ & $W_{\lambda}(\mathrm{m} \AA)$ & $\log (\mathrm{Hg} / \mathrm{H})$ \\
\hline Hg I & 4358.32 & 58.35 & 22 & -4.57 \\
$\mathrm{Hg}$ I & 5460.73 & 60.73 & 29 & -4.48 \\
$\mathrm{Hg}$ II & 6149.47 & 49.46 & 41 & -4.66 \\
$\mathrm{Hg}$ II & 7944.55 & 44.57 & 23 & -4.81 \\
\hline
\end{tabular}

HD 65949 is known to be a spectroscopy binary (cf. Gieseking \& Karimie 1982), but we have been unable to find absorption lines that are definitely due to the secondary. The line at $\lambda 3984.23$ is primarily due to Re II, though there may be some contribution from $\mathrm{Cr}$ II. We discuss the Re II identification in a separate section.

Figure 3 shows the wider wavelength region of $\lambda 3984$ for the 5 stars of Fig. 1.

The red $7 \mathrm{~s}-7 \mathrm{p} \mathrm{Hg}$ II lines, $\lambda \lambda 6149$ and 7944 are also present. Additionally, all three expected $\mathrm{Hg}$ I lines, $\lambda \lambda 4046$, 4358, and 5460 are present, within $0.02 \AA$ of their laboratory positions (cf. Table 1). We measured $\lambda^{*} 4046.54$ for the first of these lines. It is blended with Pt II, whose wavelength could be anywhere from 4046.44 to $4046.52 \AA$ depending on the isotopic composition (Wahlgren et al. 2000). 
Table 2. Pt II lines with minimal blending.

\begin{tabular}{|c|c|c|c|c|c|c|}
\hline $\begin{array}{l}\lambda^{*} \\
\AA\end{array}$ & $I_{\mathrm{S}}$ & $\begin{array}{c}\left(\Delta_{\mathrm{S}}\right) \\
\AA \times 100\end{array}$ & $\begin{array}{r}\left(\Delta_{198}\right) \\
\AA \times 100\end{array}$ & $\begin{array}{l}W_{\lambda} \\
\mathrm{m} \AA\end{array}$ & $\begin{array}{l}\log \\
(\mathrm{Pt} / \Sigma)\end{array}$ & Blend \\
\hline 3766.46 & 10 & 6 & -2 & $10^{\mathrm{B}}$ & & \\
\hline 3768.45 & 10 & 6 & -1 & $10^{\mathrm{B}}$ & & \\
\hline 4023.85 & 3 & 4 & 1 & 17 & -5.74 & \\
\hline 4034.21 & 5 & 4 & -1 & 23 & -5.82 & $.23 \mathrm{Fe} \mathrm{II}$ \\
\hline 4061.71 & 10 & 5 & 3 & 37 & -5.58 & $.78 \mathrm{Fe} \mathrm{II}$ \\
\hline 4105.50 & 0 & 5 & 1 & $6^{\mathrm{B}}$ & & \\
\hline 4148.33 & 5 & 3 & 2 & 17 & -5.79 & \\
\hline 4223.76 & 3 & 7 & 5 & 12 & & \\
\hline 4288.46 & 5 & 6 & 1 & 25 & & $.56 \mathrm{Fe}$ II? \\
\hline 4514.18 & 10 & 1 & -1 & 52 & -5.69 & \\
\hline
\end{tabular}

в Line is in a Balmer wing.

We derive an abundance estimate for mercury based on our temporary model, using the lines shown in Table 1 . The values are all within a factor of two of one another. A straight mean yields $\log (\mathrm{Hg} / \mathrm{H})+12.0=7.4$. Our rough calculation for $\lambda 3984$, with a three-isotope mix, achieves a fit for an abundance between 7.1 and 7.4.

A value $\log (\mathrm{El} / \mathrm{H})=7.4$ is close to the solar iron abundance, and higher by 0.75 dex than in any of the 42 stars studied by Woolf \& Lambert (1999). Dolk et al. (2003) give mercury abundances for $31 \mathrm{HgMn}$ stars including 14 in common with Woolf and Lambert. Their highest abundance is 7.0, for AV Scl (HR 89 = HD 1909).

In addition to mercury, we also identify 15 lines wholly or partially as Pt II. The stellar wavelengths are in better agreement with laboratory wavelengths if we apply double the isotope shifts given by Engleman (1989) for ${ }^{196} \mathrm{Pt}$ vs. ${ }^{194} \mathrm{Pt}$ to get wavelengths for the heaviest stable isotope, ${ }^{198} \mathrm{Pt}$ (cf. Dworetsky 1993). Table 2 gives data for 10 of the 15 lines where we think Pt II dominates the stellar feature. The first two columns give the measured stellar wavelength, and Shenstone's (1938) intensity. The entry, $\Delta_{\mathrm{S}}$, in Col. 3 is the stellar wavelength minus Shenstone's wavelength.

Column 4 gives $\Delta_{198}$, the stellar wavelength minus that for ${ }^{198}$ Pt II, using Engleman's isotope shifts. Both differences are in hundredths of an angstrom. The equivalent width is given in Col. 5, followed by the corresponding abundance based on oscillator strengths from Dworetsky et al. (1984). Possible blends are listed in the final column. The platinum abundance excess is $\approx 4.6$ dex.

Note that Pt II is commonly identified in the cooler $\mathrm{HgMn}$ stars, and that a dominant heavy isotope ( $\mathrm{Pt}$, or $\mathrm{Hg}$ ) is also more typical of the cooler stars.

We find other similarities to HgMn stars. Both S II and P II are well identified. These ionic spectra appear sometimes individually, and sometimes together in HgMn stars. P II is not commonly identified in the magnetic sequence of CP stars. We find no evidence for Ga II, which often is found in stars with strong P II.

\section{The Re II spectrum}

We found highly significant coincidences with classified Re II lines having intensities of 20 or greater (Meggers et al. 1958). After noting that the Meggers et al. paper included a line at $3984.28 \AA$, with intensity 10 , we tested significances with an independent list of 19 lines, all with intensity 10 . The results were again highly significant. Moreover, the additional lines with intensities 10 and 15 filled in a number of previously unidentified wavelengths.

We were able to identify additional weak features with rhenium, probably Re II, with the help of Meggers's (1952) description of the arc and spark spectrum of the element. Thus far, we have identified nearly 60 lines as due wholly or partially to rhenium. A detailed listing may be found in the online material.

The Re II spectrum has been identified in CP stars, but with difficulty, and some uncertainty. Palmeri et al. (2005) give details, as well as a few oscillator strengths. The strongest Re II line for which they give an oscillator strength is $\lambda 3742.28$, $\log (g f)=-1.7$. The equivalent width of $\lambda^{*} 3742.30$ is $74 \mathrm{~m} \AA$. If the line is unblended, a provisional abundance estimate yields $\log (\operatorname{Re} / \mathrm{H})+12 \approx 6.2$., (using $\xi_{\mathrm{t}}=2 \mathrm{ks}^{-1}$ to simulate hyperfine structure). This is somewhat lower than the mercury abundance $(\approx 7.4)$. The transition probability is given only to 1 figure; the overall calculation is quite rough.

\section{Relation to the silicon stars}

HD 65949 has a weak magnetic field, and therefore some similarity to the CP2 stars. Its temperature puts it in the range of the silicon stars, but the silicon lines themselves are not particularly strong (cf. Fig. 1).

Among the known silicon stars with temperatures near $14000 \mathrm{~K}$, we might compare it with HD 200311, a mild silicon star studied by Adelman (1974). HD 200311 has sharp spectral lines, and shows $\mathrm{Hg}$ II $\lambda 3984$, for which we measured equivalent widths of 69 and $90 \mathrm{~m} \AA$ on two analog tracings kindly made available by Dr. Adelman.

The $\lambda 3984$ line of $\mathrm{Hg}$ II is identified in a number of silicon stars (cf. Albacete-Colombo et al. 2002).

HD 200311 has too strong Co II lines for it to be considered a typical CP2 star, but in any case its myriad Ce II lines set it clearly apart from HD 65949, which has no securely identified lines of the second spectrum of any lanthanide.

Another hot silicon star with published equivalent width measurements is HR 1728 (HD 34452, Tomley et al. 1970). The spectrum has substantial rotational broadening which may have obscured the $\mathrm{Hg}$ II or most lines, but Eu II $\lambda \lambda 3930$ and 4205 have measured equivalent widths of 80 and $120 \mathrm{~m} \AA$. The Si II lines themselves, are much stronger than in HD 65949.

\section{The outliers: HR 6000 and HR 6870}

The silicon-weak star HR 6000 is currently subject to a detailed study by Castelli (2006). She has used a temperature of $12850 \mathrm{~K}$, close to that appropriate for HD 65949. However, Fig. 1 shows the most extraordinary weakness of the typically strong Si II lines $\lambda \lambda 4128$ and 4130 . Given the ubiquity and common strength of these lines for stars in this temperature range, we conclude that HR 6000 is significantly different from HD 65949 and its congeners at this temperature.

Another unusual star with a comparable temperature is HD 6870 (HD 168733), noted by Bidelman \& Aller (1963). Little (1974) and Muthsam \& Cowley (1984) determined abundances. These workers noted unusually strong Cl II, and Ga II, and Ti II and III. The iron abundance is quite high. Muthsam and Cowley noted the presence of the third spectrum of $\mathrm{Nd}$. A new study in progress, of UVES spectra extending to $3048 \AA$, shows that Dy III, Sm III, and most unusually, Ce III are clearly present. 
While HD 64949 has some features in common with HR 6870, the spectra are quite different.

\section{Marginal identifications}

We analyzed 2264 wavelength measurements by the method of wavelength coincidence statistics. The survey employed 416 lists of atomic and ionic lines with various strengths. The number of spurious marginal coincidences to be expected may be determined by a Monte Carlo method. We expect between 8 and 12 marginally significant results, by chance. We obtained 25 marginal significances $(0.01$ to 0.05$)$ for the following spectra: He I, N II, Ne I (2 lists), Sc II (2 lists), Ti II, V I, Co I, Ni II (2 lists), Cu II, Kr II (2 lists), Sb II, Nd III (2 lists), Tb III, Dy III, $\mathrm{Au}$ II (3 lists), Hg II, Pu II, and Cm II. Several of these species are surely present (e.g. He I, and Nd III).

The following spectra showed highly significant coincidences (<0.001): Re II (2 lists), Os II, Te II (2 lists), Ru II, and $\mathrm{Nb}$ II (2 lists). These spectra are rarely identified in stars and are of great interest.

\section{Discussion}

HD 65949 is one of a number of CP stars in the cluster NGC 2516, which is slightly older than the Plieades. It is unclear how this fact might be related to the peculiarities discussed here.

Although HD 65949 probably has the highest mercury abundance of any known CP star, it is not a mercury-manganese star. Typically, HgMn stars with similar temperature have the greatest overabundances of manganese. Whatever mechanisms operate to produce the overabundances of the heaviest elements, have failed to produce the usual anomalies near the iron peak.

Clues to the unusual nature of HD 65949 may lie hidden among the abundances of trace and marginally identified species for which superior observational material is required.

Acknowledgements. This research has made use of the SIMBAD database, operated at CDS, Strasbourg, France. We acknowledge use of the Vienna Atomic Database (VALD).

We thank Dr. S. J. Adelman for tracings of HD 200311, and B. Smalley for a copy of code to determine stellar parameters. Dr. Luca Sbordone kindly helped
CRC port a Linux Atlas 9 to a pc running Digital Visual Fortran. C.R.C. thanks Dr. Jon Miller for a helpful discussion of the X-ray sources in NGC 2516.

\section{References}

Abt, H. A., \& Morgan, W. W. 1969, AJ, 74, 813

Adelman, S. J. 1974, ApJS, 28, 51

Albacete-Colombo, J. F., López-García, Z., Levato, H., Malaroda, S. M., \& Grosso, M. 2002, A\&A, 392, 613

Bidelman, W. P., \& Aller, L. H. 1963, AJ, 68, 273

Castelli, F. 2006, web site http://wwwuser .oats.ts.astro.it/castelli/ Cowley, C. R. 1995, in Laboratory and Astronomical High Resolution Spectra, ed. A. J. Sauval, R. Blomme, \& N. Grevesse, ASP Conf. Ser., 81, 467

Damiani, F., Flaccomio, E., Micela, G., et al. 2003, ApJ, 588, 1009

Dworetsky, M. M. 1993, in Peculiar versus Normal Phenomena in A-type and Related Stars, ed. M. M. Dworetsky, F. Castelli, \& R. Faraggiana, IAU Coll., 138,1

Dworetsky, M. M. 2005, in The A Star Puzzle, IAU Symp., 224, FP17, ed. J. Zverko, J. Žižňovský, S. J. Adelman, \& W. W. Weiss [arXiv: astro-ph/0407528]

Dworetsky, M. M., Storey, P. J., \& Jacobs, J. M. 1984, Phys. Scr., T8, 39

Dolk, L., Wahlgren, G. M., \& Hubrig, S. 2003, A\&A, 402, 299

Engleman, R. Jr. 1989, ApJ, 340, 1140

Gieseking, F., \& Karimie, M. T. 1982, A\&AS, 49, 497

Hubrig, S., North, P., Schöller, M., \& Mathys, G. 2006, AN, 327, 289

Kupka, F., Ryabchikova, T. A., Piskunov, N. E., Stempels, H. C., \& Weiss, W. W. 2000, Baltic Astron., 9, 590

Little, S. J. 1974, ApJ, 193, 639

Meggers, W. F. 1952, J. Res. NBS, 49, 187

Meggers, W. F., Catalán, M. A., \& Sales, M. 1958, J. Res. Nat. Bur. Stds., 61, 441

Moon, T. T. 1984, Comm. Univ. London Obs., No. 78

Moon, T. T., \& Dworetsky, M. M. 1985, MNRAS, 217, 305

Moore, C. E. 1945, Cont. Princeton Univ. Obs., 20

Morgan, W. W., Abt, H. A., \& Tapscott, J. W. 1978, Revised MK Spectral Atlas for Stars Earlier Than the Sun, Yerkes Observatory, University of Chicago and Kitt Peak National Observatory

Muthsam, H., \& Cowley, C. R. 1984, A\&A, 130, 348

Palmeri, P., Quinet, P., Biémont, É, Xu, H. L., \& Svanberb, S. 2005, MNRAS, 362,1348

Preston, G. W. 1974, ARA\&A, 12, 257

Shenstone, A. G. 1938, Phil. Trans. Roy. Soc. London, A237, 453

Sung, H., Bessell, M. S., Lee, B.-W., \& Lee, S.-G. 2002, AJ, 123, 290

Tomley, L. J., Wallerstein, G., \& Wolff, S. C. 1970, A\&A, 9, 380

Wahlgren, G. M., Dolk, L., Gavriele, K., Johansson, S., \& Litzén, U. 2000, ApJ, 539, 908

White, R. E., Vaughan, A. H., Jr., Preston, G. W., \& Swings, J. P. 1976, ApJ, 204, 131

Woolf, V. M., \& Lambert, D. L. 1999, ApJ, 521, 414 